\title{
Cell fate decisions are specified by the dynamic ERK interactome
}

\author{
Alex von Kriegsheim ${ }^{1}$, Daniela Baiocchi ${ }^{\# 1,6}$, Marc Birtwistle ${ }^{\# 1,8}$, David Sumpton1, Willy \\ Bienvenut $^{1}$, Nicholas Morrice ${ }^{2}$, Kayo Yamada ${ }^{3}$, Angus Lamond ${ }^{3}$, Gabriella Kalna ${ }^{1}$, Richard \\ Orton $^{5}$, David Gilbert ${ }^{4,7}$, and Walter Kolch ${ }^{1,5,8,10}$ \\ ${ }^{1}$ Signalling and Proteomics Laboratory, The Beatson Institute for Cancer Research, Glasgow G61 \\ 1BD, UK \\ ${ }^{2}$ MRC Protein Phosphorylation Unit, University of Dundee, Dundee DD1 5EH, Scotland, UK \\ ${ }^{3}$ Wellcome Trust Centre for Gene Regulation and Expression, University of Dundee, Dundee DD1 \\ $5 \mathrm{EH}$, Scotland, UK \\ ${ }^{4}$ Bioinformatics Research Centre, Department of Computing Science, University of Glasgow, \\ Glasgow G12 8QQ, UK \\ ${ }^{5}$ Sir Henry Wellcome Functional Genomics Facility, University of Glasgow, Glasgow G12 8QQ, \\ UK \\ \# These authors contributed equally to this work.
}

\section{Abstract}

Extracellular signal-regulated kinase (ERK) controls fundamental cellular functions, including cell fate decisions ${ }^{1,2}$. In PC12, cells shifting ERK activation from transient to sustained induces neuronal differentiation ${ }^{3}$. As ERK associates with both regulators and effectors ${ }^{4}$, we hypothesized that the mechanisms underlying the switch could be revealed by assessing the dynamic changes in ERK-interacting proteins that specifically occur under differentiation conditions. Using quantitative proteomics, we identified 284 ERK-interacting proteins. Upon induction of differentiation, 60 proteins changed their binding to ERK, including many proteins that were not known to participate in differentiation. We functionally characterized a subset, showing that they regulate the pathway at several levels and by different mechanisms, including signal duration, ERK localization, feedback, crosstalk with the Akt pathway and differential interaction and phosphorylation of transcription factors. Integrating these data with a mathematical model confirmed that ERK dynamics and differentiation are regulated by distributed control mechanisms rather than by a single master switch.

(C) 2009 Macmillan Publishers Limited. All rights reserved.

${ }_{6}^{10}$ Correspondence should be addressed to W.K. (walter.kolch@ucd.ie)..

${ }^{6}$ Current address: Experimental Pathology, University of Bologna, 40126 Bologna, Italy.

${ }^{7}$ Current address: School of Information Systems, Computing \& Mathematics, Brunel University, Uxbridge, Middlesex, UB8 3PH, UK.

${ }^{8}$ Current address: Systems Biology Ireland, University College Dublin, Dublin 4, Ireland.

AUTHOR CONTRIBUTIONS

A.v.K. performed most of the experiments, analysed the data and wrote the paper; D.B. performed the PEA-15 experiments; M.B., R.O. and D.G. generated the mathematical model; D.S., W.B., A.v.K., N.M., K.Y., and A.L. generated and analysed MS data; G.K. performed bioinformatic analysis; W.K. conceived the study and edited the paper.

Note: Supplementary Information is available on the Nature Cell Biology website.

COMPETING FINANCIAL INTERESTS

The authors declare no competing financial interests. 
The basic biochemistry of the ERK pathway is well known: receptors activate Ras, which recruits Raf kinases to the cell membrane for activation. Raf phosphorylates and activates MEK (mitogen-activated or extracellular signal-regulated protein kinase), which in turn phosphorylates and activates ERK ${ }^{5}$. However, how this pathway achieves different yet specific biological responses remains unclear. ERK interacts with $>170$ proteins including many substrates ${ }^{4}$. The fidelity of substrate phosphorylation is mediated by a combination of a consensus phosphorylation sequence $\mathrm{PXS} / \mathrm{TP}^{6}$ and distinct interaction motifs ${ }^{7}$. Thus, specific dynamic changes of ERK interactors in response to distinct stimuli may influence substrate specificity and biological outcomes. In PC12 cells, EGF (epidermal growth factor) stimulates transient ERK activation and cell proliferation, whereas NGF (nerve growth factor) induces sustained ERK activation and cell differentiation ${ }^{3}$. Recent studies, combining mathematical modelling with biological experimentation ${ }^{8,9}$, came to differing conclusions on what determines ERK signalling dynamics. We reasoned that a systematic comparison of differential dynamic changes in ERK-interacting proteins could provide new insights on a systems level.

We used stable isotope labelling with amino acids in cell culture (SILAC) ${ }^{10}$ to identify dynamic changes of endogenous ERK signalling complexes in PC12 cells upon stimulation with EGF and NGF (Fig. 1a). We analysed protein levels at two time points: 5 min, when ERK phosphorylation peaks in response to both ligands, and $30 \mathrm{~min}$, when ERK phosphorylation has returned to basal levels in EGF-treated cells, but is sustained in NGFstimulated cells (Fig. 1d). Statically-interacting proteins were identified by comparing ERK1 immunoprecipitates with control immunoprecipitates (Supplementary Information, Table S1). By summarizing all proteins that specifically changed in response to growth factor treatment or were different between ERK1 and control immunoprecipitations, we identified 284 proteins as specific components of endogenous ERK1 complexes (Supplementary Information, Table S2). They included known and many unknown binding partners (Supplementary Information, Table S3). Although we used an ERK1 antibody for technical reasons, all the proteins tested also interacted with ERK2 in co-immunoprecipitation assays. Thus, our results probably represent an ERK1/2 interactome. 149 of the proteins contained ERK-phosphorylation or ERK-binding motifs and were particularly enriched in D domains (Supplementary Information, Table S4). We counted 232 proteins in at least one time point and 135 proteins in both the $5 \mathrm{~min}$ and 30 min timepoints (Supplementary Information, Fig. S1 and Tables S2, S5, S6). Of the interacting proteins, 143 showed a $>1.3$ fold change in association, in at least one time point. This cut-off distinguished real changes from experimental variations (Supplementary Information, Figs S2, S3). After 5 min of NGF stimulation, only a small set of proteins was differentially associated compared with $5 \mathrm{~min}$ of EGF stimulation (Fig. 1b). Clustering NGF-specific interactions by gene ontology functions revealed an over-representation of proteins involved in transcription and regulation of gene expression (Supplementary Information, Table S7). After $30 \mathrm{~min}$, differential changes increased (Fig. 1c) and were enriched in proteins regulating transcription, differentiation/cell death, transport/localization and metabolic enzymes (Supplementary Information, Table S8). Thus, both the specificity and kinetics of ERK association were differentially regulated by growth factors. We verified the interaction profiles of 12 ERK-binding proteins using endogenous co-immunoprecipitation experiments, which showed good correlation with SILAC quantifications and no unspecific co-immunoprecipitations (Figs 1d Supplementary Information, Fig. S4).

We functionally characterized several interactors in more detail. Proteins with interactions differentially affected by EGF and NGF at 30 min are likely candidates for influencing ERK-activation dynamics. One such protein, NF1 (neurofibromin 1), is a Ras-GAP (Ras GTPase-activating protein) that is mutated in neurofibromatosis ${ }^{11}$. NGF dissociated the ERK-NF1 complex more efficiently and for longer than EGF (Fig. 1d). Neither EGF nor 
NGF affected the catalytic activity of NF1 (Supplementary Information, Fig. S5), only its interaction with Ras. Under conditions of serum starvation, exogenously expressed H-Ras formed a complex with endogenous NF1 and ERK (Fig. 2a). As the interaction between $\mathrm{NF} 1$ and Ras is unstable ${ }^{12}$, we included a chemical crosslinker to preserve the complex during immunoprecipitation. Upon EGF stimulation, NF1 dissociated from H-Ras, but reassociated after $30 \mathrm{~min}$. In contrast, NGF dissociated the complex, with no re-binding of NF1. This mirrors NF1 binding kinetics to ERK (Fig. 1d), suggesting that ERK is the NF1 binding partner that controls NF1 recruitment to Ras. The MEK inhibitor U0126 blocked stimulation-induced ERK-NF1 dissociation (Fig. 2b), indicating that ERK activity is required. NF1 phosphorylation on an ERK consensus motif was also U0126-sensitive and was induced more strongly by NGF than EGF (Fig. 2c), suggesting a positive feedback from ERK to Ras via the phosphorylation and dissociation of NF1. Unfortunately, we could not perform mutational analysis as no full-length $N F 1$ cDNA was available.

The exact role of Ras in ERK activation and PC12 cell differentiation remains unclear. A recent model suggests that Ras causes the transient activation of ERK whereas Rap1 mediates the sustained activation ${ }^{13}$. In our experiments, EGF induced transient activation of Ras, while NGF caused more sustained activation (Fig. 2d), which correlated with sustained ERK activation (Fig. 1d). Dominant-negative H-Ras ${ }^{\mathrm{N} 17}$ completely blocked ERK activation in response to EGF and NGF (Supplementary Information, Figs. S6A, B). Similarly, HRas $^{\mathrm{N} 17}$ completely inhibited NGF-stimulated Ras activation, but not Rap1a activation (Supplementary Information, Fig. S6C). Depletion of NF1 by short interfering RNA (siRNA) augmented and extended Ras, MEK and ERK activation upon EGF stimulation (Fig. 2e, f). NF1 downregulation also enhanced NGF-mediated Ras and ERK activation without affecting Rap1 activation (Supplementary Information, Fig. S6C). These results confirm a key role for Ras in shaping ERK response kinetics and suggest that NF1 is a crucial Ras regulator, in PC12 cells. Importantly, NF1 downregulation enabled EGF to partially promote PC12 cell differentiation (Fig. 2g).

PEA-15 is a multifunctional protein that does not affect ERK activation, but interferes with ERK nuclear import and its effects on gene transcription ${ }^{14}$. NGF induced a sustained dissociation of PEA-15 from ERK, whereas EGF only achieved a transient dissociation (Fig. 1d). Nuclear ERK accumulation is associated with NGF-induced differentiation ${ }^{15}$. Knocking down PEA-15 using siRNA increased ERK nuclear localization (Fig. 3a), and permitted some differentiation by EGF (Fig. 3b). PEA-15 dissociation from ERK involves phosphorylation of Ser 104 and Ser 116 (ref. 16). As Ser 116 is phosphorylated by Akt ${ }^{17}$, we tested whether PEA-15 mediates crosstalk between the ERK and Akt pathways. NGF activation of Akt is stronger and more sustained than that by EGF (Supplementary Information, Fig. S7A). Akt is downstream of PtdIns(3)K (phosphoinositide-3 kinase). A PtdIns(3)K inhibitor, LY294002, diminished ERK nuclear translocation induced by NGF (Fig. 3c), and abolished phosphorylation of Ser 116 (Fig. 3d), but had little effect on ERK activation (Supplementary Information, Fig. S7B). A S116A mutation enhanced PEA-15 binding to ERK in starved cells, and mimicked the inhibitory effect of LY294002 on NGFinduced PEA-15 dissociation from ERK (Fig. 3e). However, PEA-15S116A still partially dissociated, indicating the involvement of additional mechanisms. PEA-15 $\mathrm{S} 116 \mathrm{~A}$ overexpression inhibited differentiation to a similar extent as LY294002 (Fig. 3f, g), confirming that PEA-15 phosphorylation has a critical role in mediating PtdIns(3)K effects on differentiation.

PEA-15 was also reported to be phosphorylated by PKC (protein kinase C) on Ser 104 (ref. 18). Therefore, we examined whether Ser 104 phosphorylation mediates crosstalk between PKC and ERK. Surprisingly, neither a PKC inhibitor, Goe7874, nor LY294002 blocked Ser 104 phosphorylation. In contrast, the MEK inhibitor U0126 or a kinase negative ERK2 
mutant reduced Ser 104 phosphorylation and ERK-PEA-15 dissociation (Supplementary Information, Fig. S8A-C). The Ser 104A and Ser 116A mutations prevented the disruption of the PEA-15-ERK complex to a similar degree (Supplementary Information, Fig. S8D), and reduced differentiation more strongly than wild-type PEA-15 (Supplementary Information, Fig. S8E). Thus, disruption of the PEA-15-ERK complex is part of the differentiation programme and is regulated by crosstalk with Akt and feedback phosphorylation by ERK.

The above data also confirm that ERK nuclear translocation is necessary for differentiation, but little is known about the nuclear ERK targets involved. In SILAC experiments, a prominent stimulation-induced nuclear ERK interactor was the ERF transcription factor. ERF suppresses Ets2-mediated transcription, but ERK phosphorylation expels ERF from the nucleus, alleviating its negative effects on gene expression ${ }^{19}$. Whereas both NGF and EGF induced ERF phosphorylation, as observed by a gel shift (Fig. 4a), only NGF caused stable ERK-ERF binding (Fig. 1d). All ERF bound to ERK was phosphorylated (Supplementary Information, Fig. S9A). Interestingly, growth factors exclusively induced cytosolic ERF phosphorylation, whereas nuclear ERF always appeared unphosphorylated. NGF, but not EGF, triggered ERF export from the nucleus after $1 \mathrm{~h}$ (Fig. 4a). An ERF mutant, where all seven ERK-phosphorylation sites were removed, halved NGF-mediated differentiation (Fig. $4 b)$. These results show that differentiation requires sustained ERK activation and ERF phosphorylation, to remove ERF from the nucleus. As ERK shuttles between the nucleus and cytosol ${ }^{20,21}$, the NGF-induced sustained ERF-ERK association suggests that ERK may serve as carrier to export ERF from the nucleus.

Another transcriptional regulator we examined further was TRPS1, which represses GATA transcription $^{22}$. GATA transcription factors are crucial for differentiation of haematopoietic cells, but also of other cell types ${ }^{23,24}$. Mutations or inactivation of human TRPS1 causes tricho-rhino-phalangeal syndromes types I and III, which are characterized by symptoms (craniofacial and skeletal abnormalities, and reduced hair growth) ${ }^{25,26}$ that resemble those of cardio-facio-cutaneous syndrome, which is caused by mutations that hyperactivate the ERK pathway ${ }^{10,27}$. TRPS1 contains two ERK consensus phosphorylation motifs, which became phosphorylated in response to EGF and NGF, with NGF causing stronger and more sustained phosphorylation (Fig. 4c). Phosphorylation was MEK dependent (Fig. 4d). Mutation of the two ERK consensus site serines to alanines impaired NGF-induced TRPS1 phosphorylation and association with ERK (Fig. 4e). Overexpression of TRPS1 had little effect on NGF-induced differentiation, whereas the TRPS1 phosphorylation site mutant profoundly inhibited differentiation (Fig. 4f). Conversely, knocking down TRPS1 using siRNA increased NGF-driven differentiation (Supplementary Information, Fig. S9B). Additionally, mutant TRPS1 blocked NGF-induced transcription of a GATA responsive promoter (Fig. 4g). These data show that ERK-dependent TRPS1 phosphorylation is crucial for NGF-mediated differentiation and GATA-driven transcription.

Collectively, these results suggested that differentiation, and the underlying regulation of ERK activation kinetics and localization is distributed over the whole signal transduction network, rather than focused on a single step. In this case, a systematic perturbation of all the nodes in the network should produce many distributed effects as opposed to one or a few perturbations having dominant effects. Such a systematic perturbation study is possible by building a mathematical model and performing sensitivity analysis. Sensitivity analysis consists of perturbing the kinetic parameters characterizing the model, and observing in silico the impact which these parameter changes have on features of interest, which in this case were ERK activity at 30 min (kinetics) and the cumulative nuclear ERK activity (localization). The model developed for this task (Fig. 5a) is a kinetic model based on ordinary differential equations (Supplementary Information, Table S9; Matlab code 
provided in Supplementary Information, Data). The model is consistent with previous studies $^{8,9}$ and incorporates interaction data from the current study. Additional data on the role of PLCY (phospholipase C-Y), feedback from ERK to Ras and MEK activation, EGF receptor internalization and degradation were derived by biochemical experimentation (Supplementary Information, Fig. S10). Simulations confirmed that the model can reproduce salient features of experimentally observed behaviour, for example, the differential NF1 association with Ras, the effects of NF1 knockdown on EGF and NGF-stimulated ERK activity, and the control of PEA-15 on nuclear ERK activity, including crosstalk from Akt (Supplementary Information, Fig. S11). Thus, the model is suitable for sensitivity analysis of EGF and NGF-mediated ERK activation and nuclear ERK activity (Fig. 5b). The $y$-axes in Fig. 5b-c represent the (absolute) fractional change in the feature of interest upon a $1 \%$ change in the respective kinetic parameter value. For these $1 \%$ changes, a sensitivity coefficient of $\sim 0.001$ is a reasonable lower bound for significance, as then (as an approximation) a large perturbation of $\sim 90-100 \%$ would change the feature of interest by $\sim 10 \%$. In the case of a master switch, a single, large sensitivity coefficient would be expected. However, this is not the case, and for both EGF and NGF a large number of the sensitivity coefficients are significant. These results support the hypothesis that control of ERK activity and localization, and therefore cell fate decisions, are the result of distributed control rather than one exerted by a single master switch. Importantly, the in silico analysis predicted that regulators of mechanistically different steps should cooperate. Indeed, the double knockdown of NF1 and PEA-15 doubled the differentiation rate in response to EGF relative to single NF1 knock-down. This corresponds to a differentiation efficiency of $\sim 70 \%$ versus NGF (Fig 5c; Supplementary Information, Fig. S12).

These data show that the decision to differentiate is an integrative process that is controlled at every step of the signalling cascade by multiple mechanisms. These include regulation of ERK activation kinetics, but also less recognized mechanisms such as crosstalk, subcellular compartmentalization and differential substrate phosphorylation. The quantitative comparison of specific changes in dynamic protein interactions in response to defined signals provides a powerful tool to identify proteins that exert the effects of these signals on biochemical and biological processes. We identified many proteins that hitherto had not been known to be involved in PC12 cell differentiation, in particular a number of transcriptional repressors. Where tested, inactivation of repressors was required for efficient differentiation, suggesting that relief of transcriptional repression is critical for ERKregulated gene expression during differentiation. Interestingly, in many cases, ERK also regulated its own regulators by phosphorylation, for example, PEA-15 and NF1. This establishes feedback circuits, which could function as bistable switches ${ }^{28}$ that convert temporally confined dynamic responses into sustained effects. Our results showing that regulation occurs by distributed control at different points in the network may seem counterintuitive for a situation where a decision on an irreversible process such as cell fate is to be made. However, a distributed mode of decision making also has advantages. First, it introduces a temporal control element into commitment. Instead of a single decision point, commitment becomes an integrator function. This is not only an efficient noise filter, but also can generate specificity of the signal to precisely determine the number of differentiating cells. Conceptually, this resembles the kinetic proofreading model of T-cell receptor signalling, which shows that accumulative activation cascades are an efficient means to discriminate between antigens that only slightly differ in affinity ${ }^{29}$. Second, it provides a 'collegial element', that is, more interfaces for integration with other processes. This is especially important for coordinating responses in the physiological context of a tissue, where cells are exposed to many growth factors simultaneously rather than to single, defined signals. There, an integrative process for deciding cell fate seems preferable to a single-switch-controlled all-or-nothing decision. 


\section{METHODS}

\section{Cells and reagents}

PC12 cells were cultured in DMEM supplemented with $2 \mathrm{mM}$ glutamine, $10 \%$ horse serum and 5\% FCS. For the SILAC experiments, the horse serum was dialysed against saline solution, and the media was supplemented with L-arginine and L-lysine, L-arginine- ${ }^{13} \mathrm{C}_{6}{ }^{14} \mathrm{~N}_{4}$ and $\mathrm{L}_{\mathrm{L}}$ lysine- ${ }^{2} \mathrm{H}_{4}$, or $\mathrm{L}$-arginine- ${ }^{13} \mathrm{C}_{6}{ }^{-15} \mathrm{~N}_{4}$ and $\mathrm{L}_{\mathrm{L}}-\mathrm{lysine}-{ }^{13} \mathrm{C}_{6}{ }^{-1}{ }^{15} \mathrm{~N}_{2}$. Plasmid and siRNA oligonucleotides were nucleofected using the manufacturer's instructions (Amaxa Biosystems). Flag-PEA-15, HA-H-Ras and Flag-H-Ras ${ }^{\mathrm{N} 17}$ were provided by P. Crespo (IBBC-CSIC, Spain); ERF and ERF ${ }^{1-7 A}$ by G. Mavrothalassitis (University of Crete, Greece); TRPS1 by F. Kaiser (University of Lübeck, Germany); HA-ERK2 and kinase-dead HA-ERK2 (ATP binding-site mutant K52R) by M. Karin (University of California, USA).

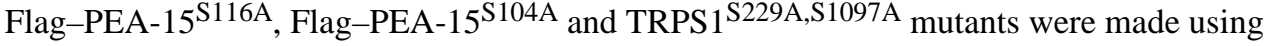
the Quickchange kit (Stratagene). Antibodies against TRPS1, ERF, RSK1-3, VGF, actin, ERK and Akt1 were from Santa Cruz; Lamin A/C, MEK 1/2, phospho-MEK1/2, Akt pSer 473, Akt, PEA-15, PEA-15 pSer 104, PLCy, phospho-PLCY (Tyr 783), and ERK substrate motif (pTP, PXpST) were from Cell Signalling; ERK1/2 and phospho-ERK1/2 were from Sigma; pan-Ras and ROCK1 were from BD-Biosciences; PEA-15 pSer 116 was from Invitrogen; $\gamma$-tubulin was from Sigma; IQGAP was from MBL; anti-MKP4 was a gift from S. Keyse (Ninewells Hospital, UK), GATA-Luciferase reporter vector was from Panomics. All primary antibodies were used at a 1:1,000 dilution, except for anti-ERF1/2 and phosphoERK1/2, which were used at a 1:500 dilution.

\section{Cell treatment and ERK immunoprecipitation}

PC12 cells were grown in SILAC medium for two weeks on collagen-coated plates. For each condition, $5 \times 10^{7}$ cells were used, after being serum-deprived for $18 \mathrm{~h}$ and treated with EGF $\left(20 \mathrm{ng} \mathrm{ml}^{-1}\right)$ or NGF $\left(100 \mathrm{ng} \mathrm{ml}^{-1}\right)$, as indicated. Cells were lysed in ice-cold lysis buffer (20 mM HEPES at pH 7.5, $150 \mathrm{mM} \mathrm{NaCl}, 1 \%$ NP40 and 2 mM EDTA) supplemented with protease ( $1 \mathrm{mM}$ phenylmethylsulfonylfluorid, $5 \mu \mathrm{g} \mathrm{ml}^{-1}$ leupeptin, 2.2 $\mu \mathrm{g} \mathrm{ml}{ }^{-1}$ aprotinin and $2 \mathrm{mM}$ sodium fluoride) and phosphatase ( $1 \mathrm{mM}$ sodium vanadate, 1 $\mathrm{mM}$ sodium pyrophosphate and $20 \mathrm{mM} \beta$-glycerophosphate) inhibitors. Lysates were centrifuged at 20,000 $\mathrm{g}$ for $10 \mathrm{~min}$, and the supernatant was pre-cleared by incubation with Protein A agarose beads (GE Healthcare) for $30 \mathrm{~min}$. For immunoprecipitation, ERK1 C16 (100 $\mu \mathrm{g}$; Santa Cruz) or rabbit pre-immune serum antibodies were covalently coupled to protein-A beads using dimethyl pimelimidate (Pierce) and $1 \mu \mathrm{l}$ of antibody per $1 \mu \mathrm{l}$ of beads. Antibody beads were washed with glycine $\mathrm{pH} 2.5$ followed by lysis buffer and resuspended in 50\% glycerol/ lysis buffer. The optimal amount of antibody beads was determined by titrating the beads in immunoprecipitation experiments against fixed amounts of lysates. For preparative purposes, antibody beads $(100 \mu \mathrm{l})$ were incubated with of lysate $(6 \mathrm{ml}$; prepared from ca. $5 \times 10^{7}$ cells) for $2 \mathrm{~h}$ at $4{ }^{\circ} \mathrm{C}$. To identify unspecifically interacting proteins, FlagM2 Agarose beads ( $20 \mu \mathrm{l}$; Sigma) were added to pre-immune beads (100 $\mu \mathrm{l})$ in the negative control. Immunoprecipitates were washed three times with $20 \mathrm{mM}$ HEPES at $\mathrm{pH} 7.5,50 \mathrm{mM}$ $\mathrm{NaCl}, 0.1 \%$ NP40, 2mM EDTA buffer supplemented with protease and phosphatase inhibitors. The immunoprecipitates of the three conditions were combined and then eluted twice with $300 \mu \mathrm{l}$ elution buffer $(200 \mathrm{mM}$ glycine at $\mathrm{pH} 2.5,500 \mathrm{mM} \mathrm{NaCl}$ and $0.1 \%$ NP40), and immediately neutralized with TrisHCl (60 $\mu \mathrm{l}$ at $\mathrm{pH} 8.9)$.

\section{Mass spectrometry}

Eluates were separated by SDS-PAGE using $10 \%$ Novex pre-cast gels. Each gel lane was cut into 8-12 slices, further dissected into $1 \times 1 \mathrm{~mm}^{2}$ fragments, dehydrated with $\mathrm{MeCN}$, reduced with $10 \mathrm{mM}$ DTT and $50 \mathrm{mM}$ ammonium bicarbonate (AB) for $20 \mathrm{~min}$ at $56^{\circ} \mathrm{C}$, alkylated with $55 \mathrm{mM}$ iodacetamide and $50 \mathrm{mM} \mathrm{AB}$ for $1 \mathrm{~h}$ at room temperature, and washed 
by sequential dehydration/hydration steps alternating between $\mathrm{MeCN}$ and $50 \mathrm{mM} \mathrm{AB}$. Then, samples were digested with trypsin (Promega) at $37^{\circ} \mathrm{C}$ for $90 \mathrm{~min}$, extracted with $0.1 \%$ trifluoroacetic acid and 5\% MeCN, and concentrated to $40 \mu \mathrm{l}$ before separation on an Ultimate 3000 nanoLC (Dionex). Next, $10 \mu \mathrm{l}$ were pre-concentrated on a PepMap100 trap column loaded at $30 \mu \mathrm{min}^{-1}$ in solvent $\mathrm{A}$ ( $5 \% \mathrm{MeCN}$ and $0.1 \%$ formic acid), and separated on a PepMap $100 \mathrm{C} 1875 \mu \mathrm{m} \times 15 \mathrm{~cm}$ analytical column using a gradient of solvent B $(80 \%$ $\mathrm{MeCN}, 0.1 \%$ formic acid; $8-25 \%$ for 70 minutes, then $25-50 \%$ for 30 minutes) at a flow rate

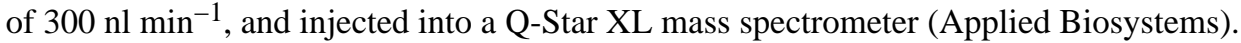
Mass spectroscopy (MS) data were acquired using a 1.5-s survey scan (mass range 400$1200 \mathrm{Da}$ ) and data-dependent MS/MS, of the three most intense ions with charge state +2 to +4 , for $2 \mathrm{~s}$. These ions were then excluded from acquisition for $30 \mathrm{~s}$. Mass spectra were recalibrated using an in-house script, searched against the IPI Rodent database (Ver. 3.32) or the rodent fraction of UniProtKB-Swiss-Prot (Ver. 54.3) using Mascot 2.0 (Matrix Science) with a mass tolerance of $12-30 \mathrm{ppm}$ for the parent and $0.12 \mathrm{Da}$ for the fragments ions. The protein identification list was filtered using the non-MUDPit scoring with a threshold of 14 (this value was determined by searching the same dataset against a randomized database). Peptides were quantified with MSQuant (http://msquant.sourceforge.net/) and subsequently normalized. Spectra were validated by visual inspection. Tryptic digests were also analysed on an LTQ-OrbiTrap (Thermo Finnigan) system as described previously ${ }^{30}$, and the peptides were quantified using MSQuant. Identified proteins were classified as specifically interacting if the ratio in both biological repeats was above 1.5 . We used 1.5 as a cut-off since the ratios were distributed as a mixture distribution with three distinct components. The first component consisted of contaminant proteins that specifically bound to the Flagcontrol immunoprecipitate; the second component contained unspecific binders that were equally precipitated by both ERK1 and control beads; and the third component contained proteins binding with a higher affinity to the ERK1 beads. The local minima of probability mass separating the components corresponded to a 1.5 fold change between the ERK1specific and unspecific proteins, which we therefore used as cut-off (Supplementary Information, Fig. S3). In Q-STAR experiments, the dynamic range was compressed. To define the cut-offs for these data, we estimated the false positive rate by plotting the ratios obtained from the true negatives, as determined by the Orbitrap experiment. 97\% of the ratios of this sample set were contained between 0.7 and 1.3 when contaminant proteins were excluded. Therefore, we classified proteins as specific ERK1 interactors if their ratio was above/below 1.3/0.7 (Supplementary Information, Fig. S4). The biological variation was small (Supplementary Information, Fig. S5)

\section{Co-immunoprecipitation experiments and western blotting}

Cells grown in standard medium were treated as above, except that less $\left(\mathrm{ca} .8 \times 10^{6}\right)$ cells were used per condition and that the antibodies were not crosslinked to beads. Immunoprecipitates were prepared as above. Proteins were eluted by boiling in SDS-PAGE sample buffer (100 mM TrisHCl at $\mathrm{pH} 6.8,2 \%$ sodium dodecylsulfate, $3 \%$ DTT), separated by SDS-PAGE and analysed by western blotting using the indicated antibodies and enhanced chemiluminescence (Roche). Rap1 and Ras activation was measured by pulldown assays with a recombinant GST-Ral-GDS or GST-Ras binding domain (RBD) protein, respectively, as described previously ${ }^{31}$. For the crosslinking experiment shown in Fig. 2a, cells were nucleofected with HA-H-Ras plasmid. Serum-starved cells were treated with 20 $\mathrm{ng} \mathrm{ml}^{-1}$ EGF or $100 \mathrm{ng} \mathrm{ml}^{-1} \mathrm{NGF}$ as indicated. Dimethyl 3,3'-dithiobispropionimidate (1mg $\mathrm{ml}^{-1}$; a cell permeable, bi-functional and cleavable crosslinker; Pierce) in PBS was added for $30 \mathrm{~min}$ at $4{ }^{\circ} \mathrm{C}$ before cells were lysed. 


\section{siRNA-mediated knockdown}

siRNA oligonucleotides ( $50 \mathrm{pmol}$ ) were introduced into PC12 cells by nucleofection (Amaxa Biosystems) according to the manufacturer's instructions. The following siRNA sequences were used: PEA-15\#1, 5'-GCAUUUACAUUUGAGCUAA-3'; \#2, 5' GCCCUAAUACACUCACCUA-3'; \#3, 5'-CGGAGAUGAUUAGAUCUAA-3' (Qiagen). Smart-pool siRNAs (Dharmacon) were used to knock-down: TRPS1 (5'GGAAACAGUUCAUCCGUAUUU-3', 5'-CGGACAAGAAAGCGCCUUAUU-3', 5' UUGCAGAAUUGAACACGAAUU-3', $5^{\prime}$-CUUCAGACUCACCCGAAUAUU-3'); $N F 1$ (5'-GAGAUGAAAUUUCGGAAUA-3', 5'-GUAACAAGCCUUAAGUUUA-3', 5' GAUAGAAGUUCCUGUCAUU-3', 5'-GAAGACGACCUUUCGAUAA-3'); $P L C \gamma\left(5^{\prime}\right.$ UAACUUGGCUCAUGGAAGA-3', 5'-UAACCGAGAGGAUCGUAUA-3', $5^{\prime}$ GAAGGACUCGGGUCAAUGG-3', 5'-GCACCGUCAUGACUUUGUU-3'). A nontargeting siRNA pool (Dharmacon) was used as control.

\section{Cell fractionation}

To separate nuclear and cytosolic fractions, cells were washed, scraped into $0.3 \mathrm{ml}$ of buffer A (50 mM $\beta$-glycerophosphate at pH 7.3, $1.5 \mathrm{mM}$ EGTA, 1 mM EDTA, $1 \mathrm{mM}$ PMSF, 2 $\mathrm{mM} \mathrm{NaF}, 0.5 \mathrm{mM}$ sodium vanadate and $1 \mu \mathrm{g} \mathrm{ml}^{-1}$ leupeptin) and centrifuged (600 $g$ for 5 min at $\left.4{ }^{\circ} \mathrm{C}\right)$. The pellet was resuspended in $200 \mu \mathrm{l}$ of lysis Buffer $(40 \mathrm{mM}$ HEPES at $\mathrm{pH}$ 7.5, $0.1 \%$ Nonidet P-40, $5 \mathrm{mM}$ EGTA, $5 \mathrm{mM} \mathrm{MgCl}_{2}$ and $1 \mathrm{mM}$ benzamidine) containing protease and phosphates inhibitors. The lysate was mixed vigorously and centrifuged $\left(13,000 \mathrm{~g}\right.$ for $5 \mathrm{~min}$ at $\left.4{ }^{\circ} \mathrm{C}\right)$ to yield a supernatant that contained the cytosolic fraction. Nuclear proteins were extracted by resuspending the pellet in $100 \mu \mathrm{l}$ extraction buffer (50 $\mathrm{mM} \beta$-glycerophosphate at $\mathrm{pH} 7.3,420 \mathrm{mM} \mathrm{NaCl}, 0.5 \mathrm{mM}$ sodium vanadate, $1.5 \mathrm{mM}$ $\mathrm{MgCl}_{2}, 0.2 \mathrm{mM}$ EDTA and 25\% glycerol). The purity of the fractions was ascertained by blotting for constitutively cytosolic (MEK) and nuclear (lamin A/C) proteins.

\section{PC12 cell differentiation assay}

PC12 cells were co-transfected with $1 \mu \mathrm{g}$ of eGFP expression plasmid (Clontech) and plasmids or siRNAs as indicated, plated on collagen-coated slides and serum-starved overnight. Cells were then stimulated with $20 \mathrm{ng} \mathrm{ml}^{-1}$ EGF or $100 \mathrm{ng} \mathrm{ml}^{-1}$ NGF. After $24 \mathrm{~h}$, cells were washed with phosphate buffered saline and fixed with $3 \%$ formaldehyde. Cells were imaged with an Olympus CKX41 microscope. GFP-positive cells were scored as differentiated if the protrusion were longer than two cell bodies.

\section{GO clustering and motif analysis}

Using DAVID tools (http://david.abcc.ncifcrf.gov/) the gene name lists were analysed and enriched ontologies were clustered using medium stringency. ERK-binding domains and ERK phosphorylation sites were identified using Scansite http://scansite.mit.edu/32. After conversion of the gene names into Swissprot denominators for human proteins, the list was batch searched under stringent criteria.

\section{Mathematical modelling}

Details are described in Supplementary Information, Data, which also contains the corresponding Matlab files.

\section{Database accession numbers}

MINT (http://mint.bio.uniroma2.it/mint/Welcome.do) accession numbers are MI-11701 and MI-0471. 


\section{Supplementary Material}

Refer to Web version on PubMed Central for supplementary material.

\section{Acknowledgments}

We thank P. Mortensen for help with MSQuant, A. Pitt and C. Ward for help with MS, O.Rath for the design of primers and G. Cesareni and A. Chatraryamontri for help with submission of the interaction data to the IMEX consortium through MINT. The work was supported by the European Union Interaction Proteome project (LSHGCT-2003-505520), the RASOR project (BBSRC and EPSRC) and Cancer Research UK.

\section{References}

1. Shaul YD, Seger R. The MEK/ERK cascade: from signaling specificity to diverse functions. Biochim. Biophys. Acta. 2007; 1773:1213-1226. [PubMed: 17112607]

2. Galabova-Kovacs G, et al. ERK and beyond: insights from B-Raf and Raf-1 conditional knockouts. Cell Cycle. 2006; 5:1514-1518. [PubMed: 16861903]

3. Marshall CJ. Specificity of receptor tyrosine kinase signaling: transient versus sustained extracellular signal-regulated kinase activation. Cell. 1995; 80:179-185. [PubMed: 7834738]

4. Yoon S, Seger R. The extracellular signal-regulated kinase: multiple substrates regulate diverse cellular functions. Growth Factors. 2006; 24:21-44. [PubMed: 16393692]

5. Leicht DT, et al. Raf kinases: function, regulation and role in human cancer. Biochim. Biophys. Acta. 2007; 1773:1196-1212. [PubMed: 17555829]

6. Clark-Lewis I, Sanghera JS, Pelech SL. Definition of a consensus sequence for peptide substrate recognition by $\mathrm{p} 44 \mathrm{mpk}$, the meiosis-activated myelin basic protein kinase. J. Biol. Chem. 1991; 266:15180-15184. [PubMed: 1907971]

7. Akella R, Moon TM, Goldsmith EJ. Unique MAP Kinase binding sites. Biochim. Biophys. Acta. 2008; 1784:48-55. [PubMed: 18068683]

8. Sasagawa S, Ozaki Y, Fujita K, Kuroda S. Prediction and validation of the distinct dynamics of transient and sustained ERK activation. Nature Cell Biol. 2005; 7:365-373. [PubMed: 15793571]

9. Santos SD, Verveer PJ, Bastiaens PI. Growth factor-induced MAPK network topology shapes Erk response determining PC-12 cell fate. Nature Cell Biol. 2007; 9:324-330. [PubMed: 17310240]

10. Niihori T, et al. Germline KRAS and BRAF mutations in cardio-facio-cutaneous syndrome. Nature Genet. 2006; 38:294-296. [PubMed: 16474404]

11. Harrisingh MC, Lloyd AC. Ras/Raf/ERK signalling and NF1. Cell Cycle. 2004; 3:1255-1258. [PubMed: 15467460]

12. Ahmadian MR, Hoffmann U, Goody RS, Wittinghofer A. Individual rate constants for the interaction of Ras proteins with GTPase-activating proteins determined by fluorescence spectroscopy. Biochemistry. 1997; 36:4535-4541. [PubMed: 9109662]

13. York RD, et al. Rap1 mediates sustained MAP kinase activation induced by nerve growth factor. Nature. 1998; 392:622-626. [PubMed: 9560161]

14. Formstecher E, et al. PEA-15 mediates cytoplasmic sequestration of ERK MAP kinase. Dev. Cell. 2001; 1:239-250. [PubMed: 11702783]

15. Traverse S, Gomez N, Paterson H, Marshall C, Cohen P. Sustained activation of the mitogenactivated protein (MAP) kinase cascade may be required for differentiation of PC12 cells. Comparison of the effects of nerve growth factor and epidermal growth factor. Biochem. J. 1992; 288(Pt 2):351-355. [PubMed: 1334404]

16. Krueger J, Chou FL, Glading A, Schaefer E, Ginsberg MH. Phosphorylation of phosphoprotein enriched in astrocytes (PEA-15) regulates extracellular signal-regulated kinase-dependent transcription and cell proliferation. Mol. Biol. Cell. 2005; 16:3552-3561. [PubMed: 15917297]

17. Trencia A, et al. Protein kinase B/Akt binds and phosphorylates PED/PEA-15, stabilizing its antiapoptotic action. Mol. Cell Biol. 2003; 23:4511-4521. [PubMed: 12808093] 
18. Araujo H, Danziger N, Cordier J, Glowinski J, Chneiweiss H. Characterization of PEA-15, a major substrate for protein kinase C in astrocytes. J. Biol. Chem. 1993; 268:5911-5920. [PubMed: 8449955]

19. Le Gallic L, Virgilio L, Cohen P, Biteau B, Mavrothalassitis G. ERF nuclear shuttling, a continuous monitor of Erk activity that links it to cell cycle progression. Mol. Cell Biol. 2004; 24:1206-1218. [PubMed: 14729966]

20. Ando R, Mizuno H, Miyawaki A. Regulated fast nucleocytoplasmic shuttling observed by reversible protein highlighting. Science. 2004; 306:1370-1373. [PubMed: 15550670]

21. Volmat V, Camps M, Arkinstall S, Pouyssegur J, Lenormand P. The nucleus, a site for signal termination by sequestration and inactivation of p42/p44 MAP kinases. J. Cell Sci. 2001; 114:3433-3443. [PubMed: 11682603]

22. Malik TH, et al. Transcriptional repression and developmental functions of the atypical vertebrate GATA protein TRPS1. EMBO J. 2001; 20:1715-1725. [PubMed: 11285235]

23. Cantor AB. GATA transcription factors in hematologic disease. Int. J. Hematol. 2005; 81:378-384. [PubMed: 16158817]

24. Brewer A, Pizzey J. GATA factors in vertebrate heart development and disease. Expert Rev. Mol. Med. 2006; 8:1-20. [PubMed: 16987437]

25. Kaiser FJ, et al. Novel missense mutations in the TRPS1 transcription factor define the nuclear localization signal. Eur. J. Hum. Genet. 2004; 12:121-126. [PubMed: 14560312]

26. Kobayashi H, et al. Missense mutation of TRPS1 in a family of tricho-rhino-phalangeal syndrome type III. Am. J. Med. Genet. 2002; 107:26-29. [PubMed: 11807863]

27. Rodriguez-Viciana P, et al. Germline mutations in genes within the MAPK pathway cause cardiofacio-cutaneous syndrome. Science. 2006; 311:1287-1290. [PubMed: 16439621]

28. Kholodenko BN. Cell-signalling dynamics in time and space. Nature Rev. Mol. Cell Biol. 2006; 7:165-176. [PubMed: 16482094]

29. George AJ, Stark J, Chan C. Understanding specificity and sensitivity of T-cell recognition. Trends Immunol. 2005; 26:653-659. [PubMed: 16236548]

30. Trinkle-Mulcahy L, et al. Identifying specific protein interaction partners using quantitative mass spectrometry and bead proteomes. J. Cell Biol. 2008; 183:223-239. [PubMed: 18936248]

31. de Rooij J, Bos JL. Minimal Ras-binding domain of Raf1 can be used as an activation-specific probe for Ras. Oncogene. 1997; 14:623-625. [PubMed: 9053862]

32. Obenauer JC, Cantley LC, Yaffe MB. Scansite 2.0: Proteome-wide prediction of cell signaling interactions using short sequence motifs. Nucleic Acids Res. 2003; 31:3635-3641. [PubMed: 12824383] 
a

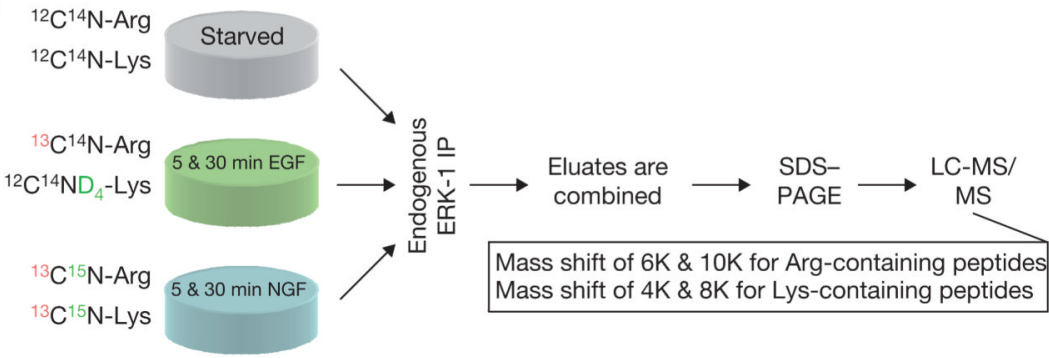

c

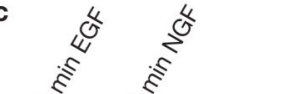

d

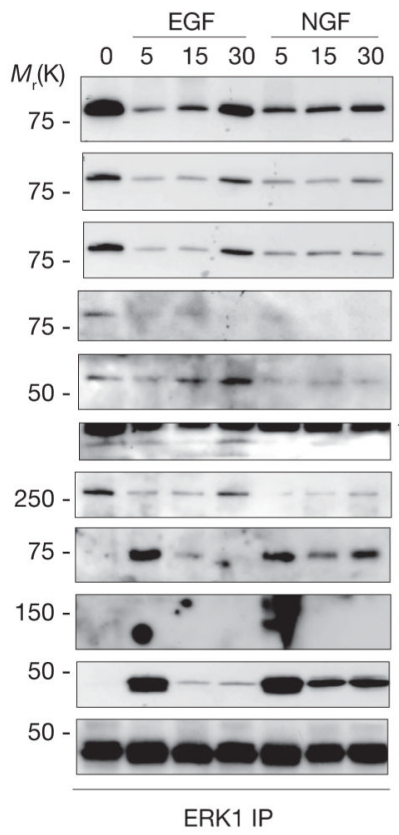

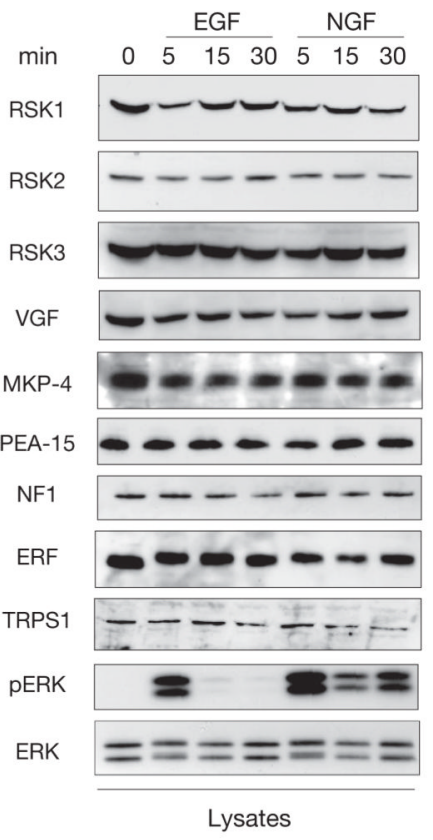

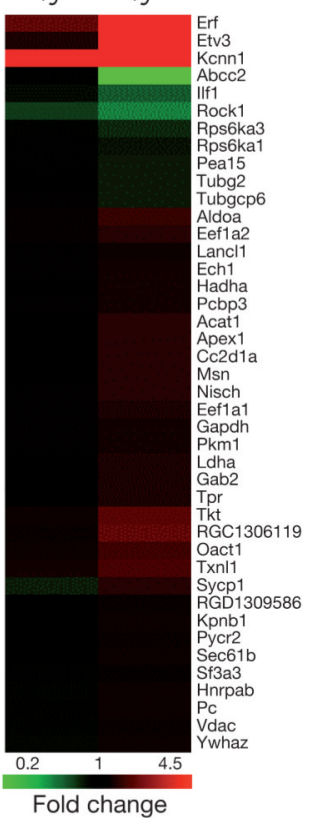

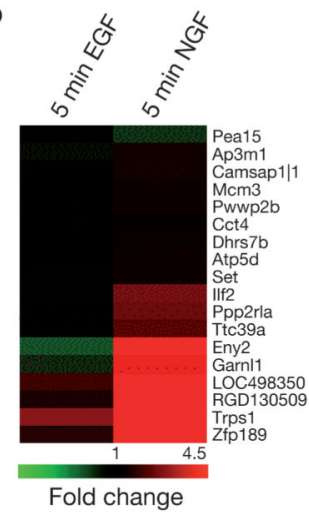

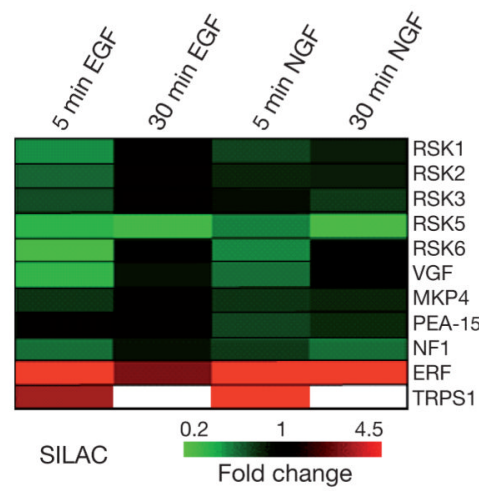

Figure 1.

Identification of dynamic ERK1 interactions. (a) Workflow of the SILAC experiments. PC12 cells were grown in media supplemented with 'light', 'heavy' and 'super heavy' arginine and lysine isotopes. The coloured isotope numbers highlight changes in residues compared with serum-starved cells. Endogenous ERK1 was immunoprecipitated from

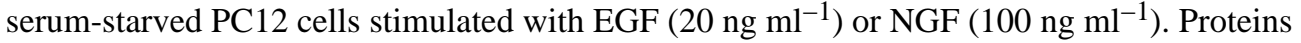
were eluted from the antibody, combined, separated by SDS-PAGE and analysed by mass spectrometry. IP, immunoprecipitate. LC-MS/MS, liquid chromatography-tandem mass spectrometry. (b, c) Heatmaps of growth factor-induced changes of proteins bound to ERK after 5 min (b) and 30 min (c) of stimulation, based on mass spectroscopy data. (d) Confirmation of selected dynamic interactions by co-immunoprecipitation and western blot analysis. PC12 cells were starved overnight and stimulated with EGF or NGF as indicated. Left panel, ERK1 immunoprecipitates were subjected to western blotting with the indicated antibodies. Asterisk indicates an unspecific band. Complete blots are shown in Supplementary Information, Fig. S17. Middle panel, total lysates from the same cells were blotted showing that protein expression remained constant during the experiment. Right panel, dynamic changes in the association to ERK1, as shown from quantified mass spectrometry data. 

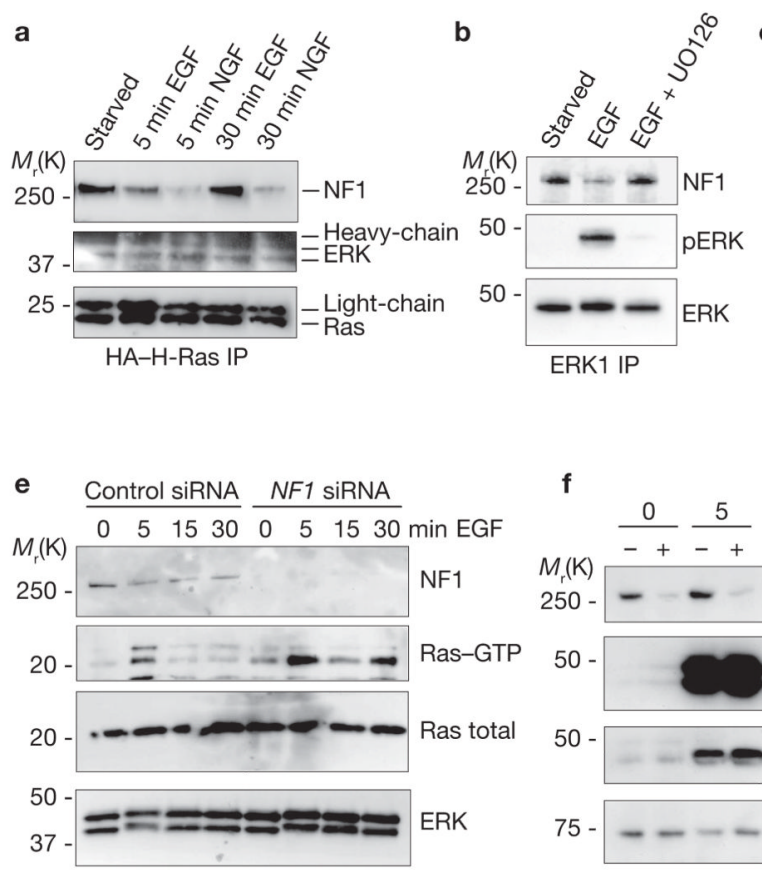

f
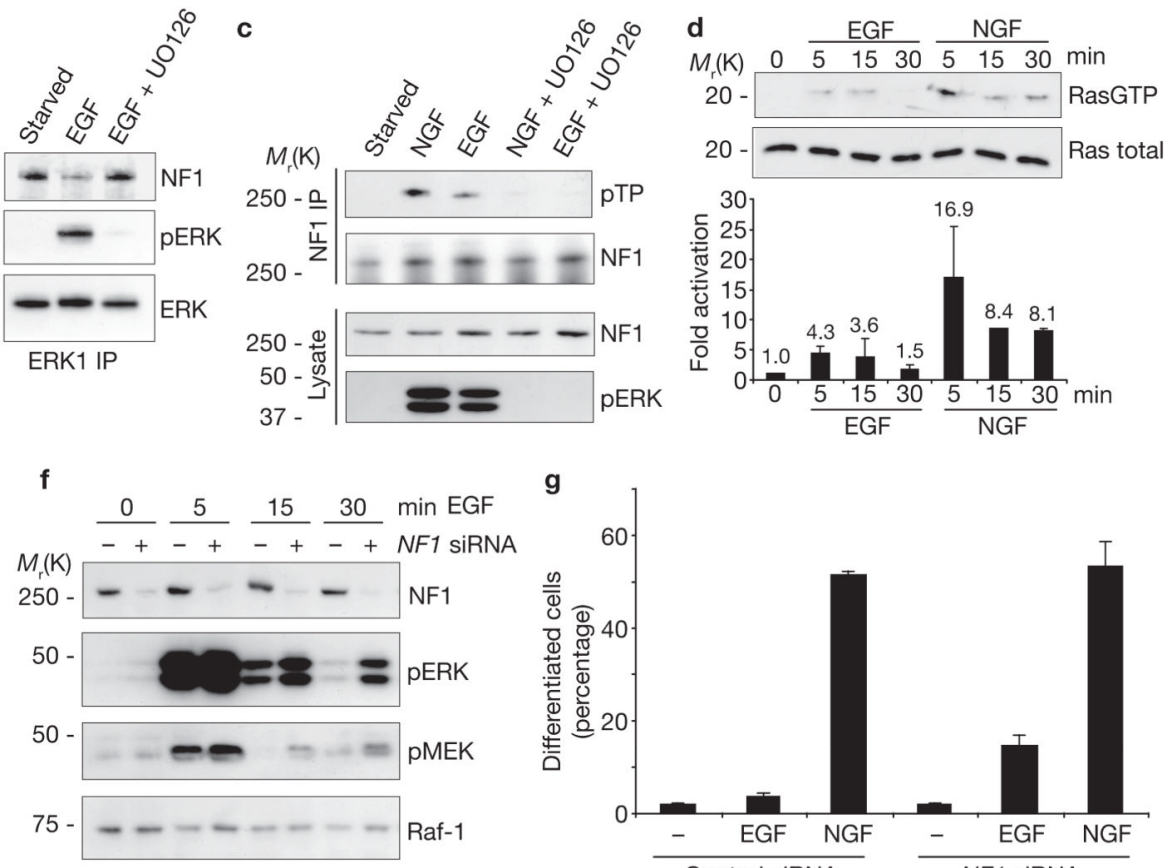

Figure 2.

NF1 controls Ras activity and signal duration. (a) PC12 cells were transfected with HA-HRas, serum-starved overnight and stimulated as indicated. After treatment with a chemical crosslinker, cells were lysed and HA-H-Ras immunoprecipitates (IP) were subjected to western blotting with the indicated antibodies. (b) ERK1 immunoprecipitates were prepared from PC12 cells treated with EGF (for $5 \mathrm{~min}$ ) or EGF and U0126 (10 $\mu \mathrm{M}$ pre-incubation for $1 \mathrm{~h}$ ), as indicated, and were examined for associated NF1. (c) NF1 immunoprecipitates from PC12 cells treated as above were blotted with an antibody specific to phospho-Thr-Pro (pTP). (d) PC12 cells were serum-starved overnight and stimulated with EGF or NGF. Activated, GTP-loaded Ras was precipitated with GST-RBD beads and subjected to western blotting with an anti-pan Ras antibody. The bar graph shows the mean \pm s.e.m. of three independent experiments. (e) PC12 cells were transfected with control or NF1 siRNA, starved overnight and stimulated with EGF. GTP-loaded Ras was precipitated with GSTRBD beads and subjected to western blotting with a pan Ras antibody. Lysates were blotted for NF1, and total Ras and ERK were used as loading controls. (f) PC12 cells were transfected with control (-) and $N F 1(+)$ siRNA and treated as above. Lysates were blotted for MEK and ERK phosphorylation, and total Raf-1 was used as a loading control. (g) PC12 cells were co-transfected with an eGFP expression vector plus control or NF1 siRNA. Serum-starved cells were stimulated with EGF or NGF. After 24 h, fluorescent eGFPexpressing cells with protrusions longer than two cell diameters were counted as differentiated. Error bars show mean \pm s.e.m. of three independent assays. 
a

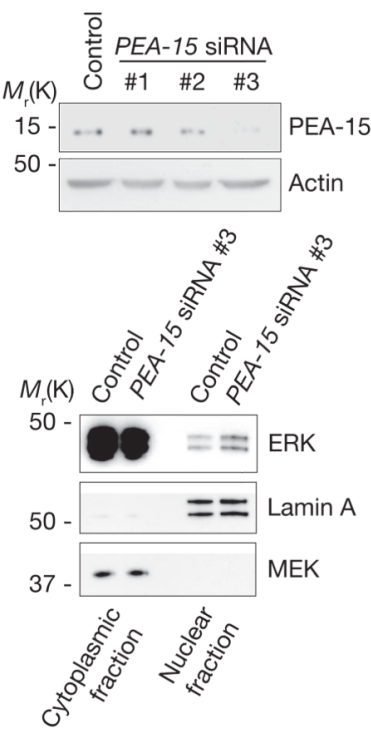

f

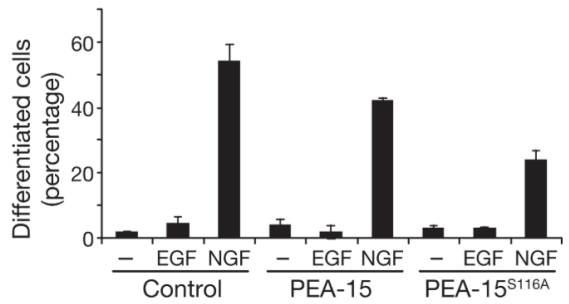

b

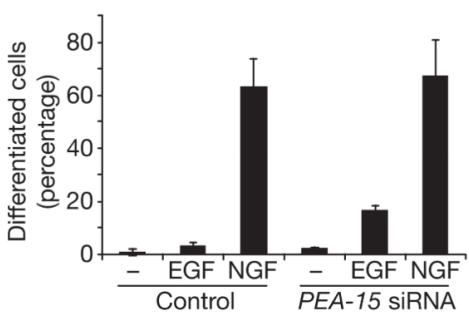

d

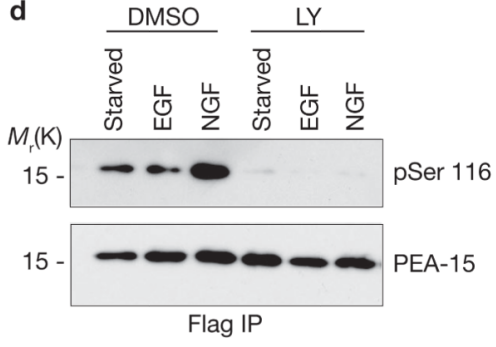

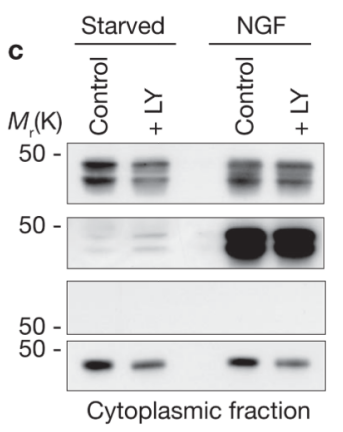

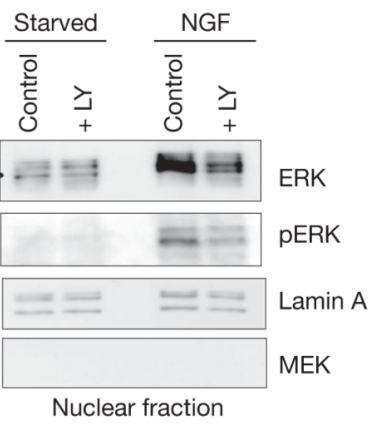

e

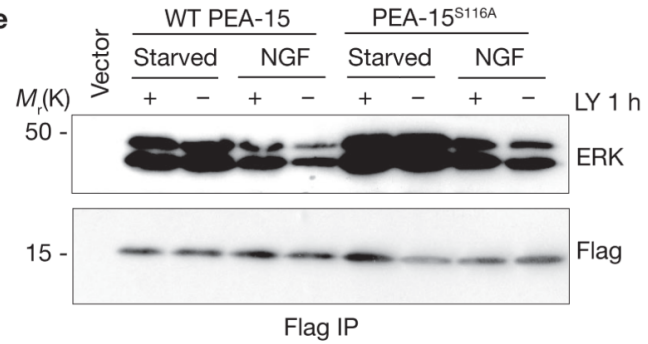

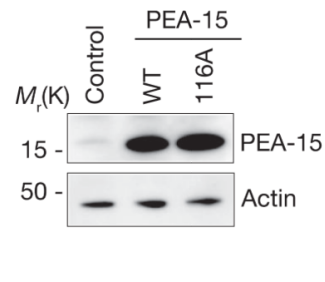

g

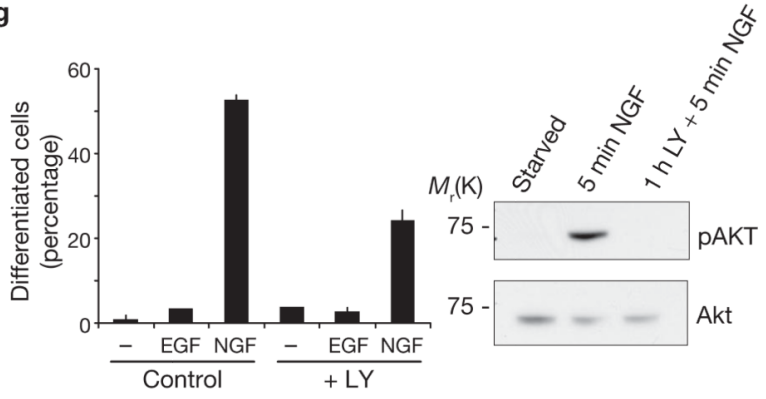

Figure 3.

Regulation of ERK localization in PC12 cells by PEA-15. (a) Top panel, cells were transfected with control and siRNAs and PEA-15 levels were examined $24 \mathrm{~h}$ later. Bottom panel, nuclear and cytosolic fractions were prepared from siRNA-transfected, serum-starved PC12 cells and subjected to western blotting with the indicated antibodies. To assess the quality of the fractionation, lysates were blotted for proteins with known nuclear (Lamin A) or cytosolic (MEK) localization. (b) Cells were co-transfected with an eGFP expression vector plus control or PEA-15 siRNA \#3. Differentiation was assessed as in Fig. 2g. (c) Serum-starved cells were treated with $10 \mu \mathrm{M}$ of PtdIns(3)K inhibitor LY294002 (LY) or DMSO (Control) for $1 \mathrm{~h}$ before stimulation with NGF for $15 \mathrm{~min}$. Nuclear and cytosolic fractions were blotted with the indicated antibodies. (d) Cells were transfected with FlagPEA-15, starved overnight and incubated with LY294002 or DMSO (Control) for $1 \mathrm{~h}$ before stimulation with EGF or NGF for $15 \mathrm{~min}$. Lysates were blotted with the indicated antibodies. (e) Cells were transfected with Flag-PEA-15 (WT) or the Flag-PEA-15 $116 \mathrm{~A}$ mutant and serum-starved overnight. Cells were pretreated with LY294002 (+) or DMSO (-) for $1 \mathrm{~h}$ and stimulated with NGF for $15 \mathrm{~min}$. Flag immunoprecipitates (IPs) were blotted for associated endogenous ERK. (f) Cells were transfected with expression plasmids for eGFP, PEA-15, PEA-15 ${ }^{\text {S116A }}$ or a vector control, and assessed for differentiation as in Fig. 2g. Expression of PEA-15 constructs was monitored by blotting with an anti-PEA-15 antibody. Error bars show the mean \pm s.e.m. of three independent experiments. (g) Cells were transfected with eGFP, serum-starved overnight, incubated with $10 \mu \mathrm{M}$ of DMSO or LY294002 for $1 \mathrm{~h}$, and subsequently stimulated with EGF or NGF for $24 \mathrm{~h}$ (left panel). 
Differentiation was scored as described in Fig. 2g. LY294002 inhibits Akt activation, as shown by western blotting of Akt phosphorylation (right panel). LY, LY294002. 

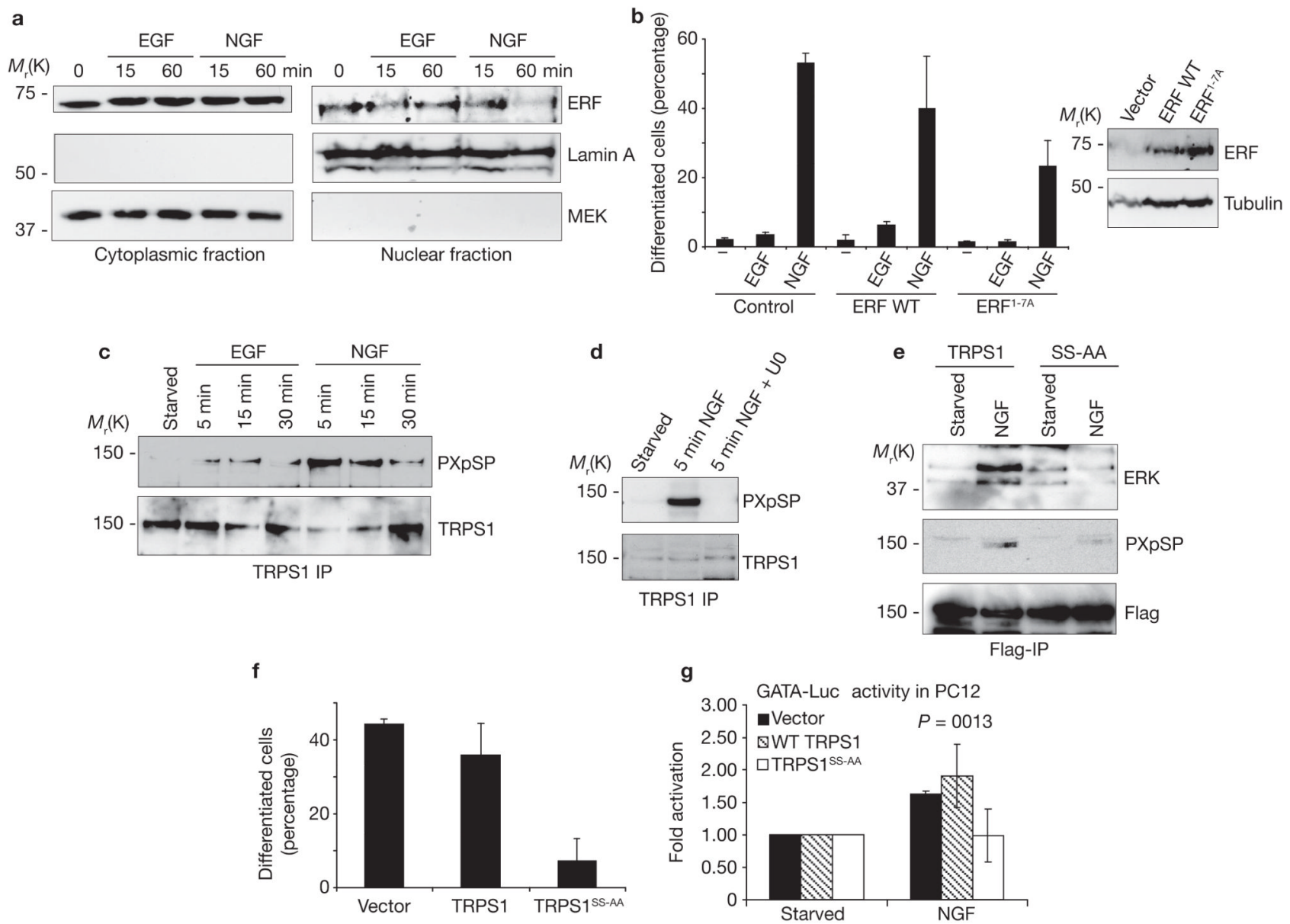

Figure 4.

Differential regulation of transcriptional repressors by EGF and NGF in PC12 cells. (a) Serum-starved cells were stimulated with EGF or NGF, as indicated. Cytoplasmic and nuclear fractions were subjected to western blotting with the indicated antibodies. (b) Cells were co-transfected with eGFP and a control plasmid, wild-type ERF or a seven serine to alanine mutant (ERF ${ }^{1-7 A}$ ), and examined for differentiation as in Fig. 2g. Equal expression of the ERF constructs was assessed for by western blotting. (c) Endogenous TRPS1 was immunoprecipitated from EGF- or NGF-treated PC12 cells and subjected to western blotting with the PXpSP antibody, which detects ERK phosphorylation sites, and a TRPS1 antibody was used as a loading control. (d) Cells were transfected with TRPS1-Flag, serum-starved overnight, then incubated for $1 \mathrm{~h}$ with DMSO or U0126 (U0) and subsequently stimulated with NGF (100 $\left.\mathrm{ng} \mathrm{m}^{-1}\right)$ for $5 \mathrm{~min}$. TRPS1-Flag immunoprecipitates were blotted with the indicated antibodies. (e) Cells were transfected with wild-type TRPS1-Flag or the S229A,S1097A mutant (SS-AA). Cells were starved overnight and stimulated with NGF for 15 min. TRPS1-Flag immunoprecipitates were blotted with the indicated antibodies. (f) Cells were co-transfected with expression vectors for eGFP and a control plasmid, TRPS1Flag or the TRPS1 ${ }^{\text {SS-AA }}$ mutant. Differentiation was assessed as in Fig. $2 \mathrm{~g}$. Error bars show the mean \pm s.e.m. of four independent experiments. (g) Cells were co-transfected with reporter vectors for GATA-firefly-luciferase, Renilla-luciferase and control plasmid, and expression vectors for TRPS1-Flag or the TRPS1 ${ }^{\text {SS-AA }}$ mutant and starved or treated with NGF (100 ng ml $\mathrm{m}^{-1}$ ) for $18 \mathrm{~h}$. The firefly luciferase output was normalized to Renilla luciferase. Error bars show the mean \pm s.e.m. of three independent experiments. 

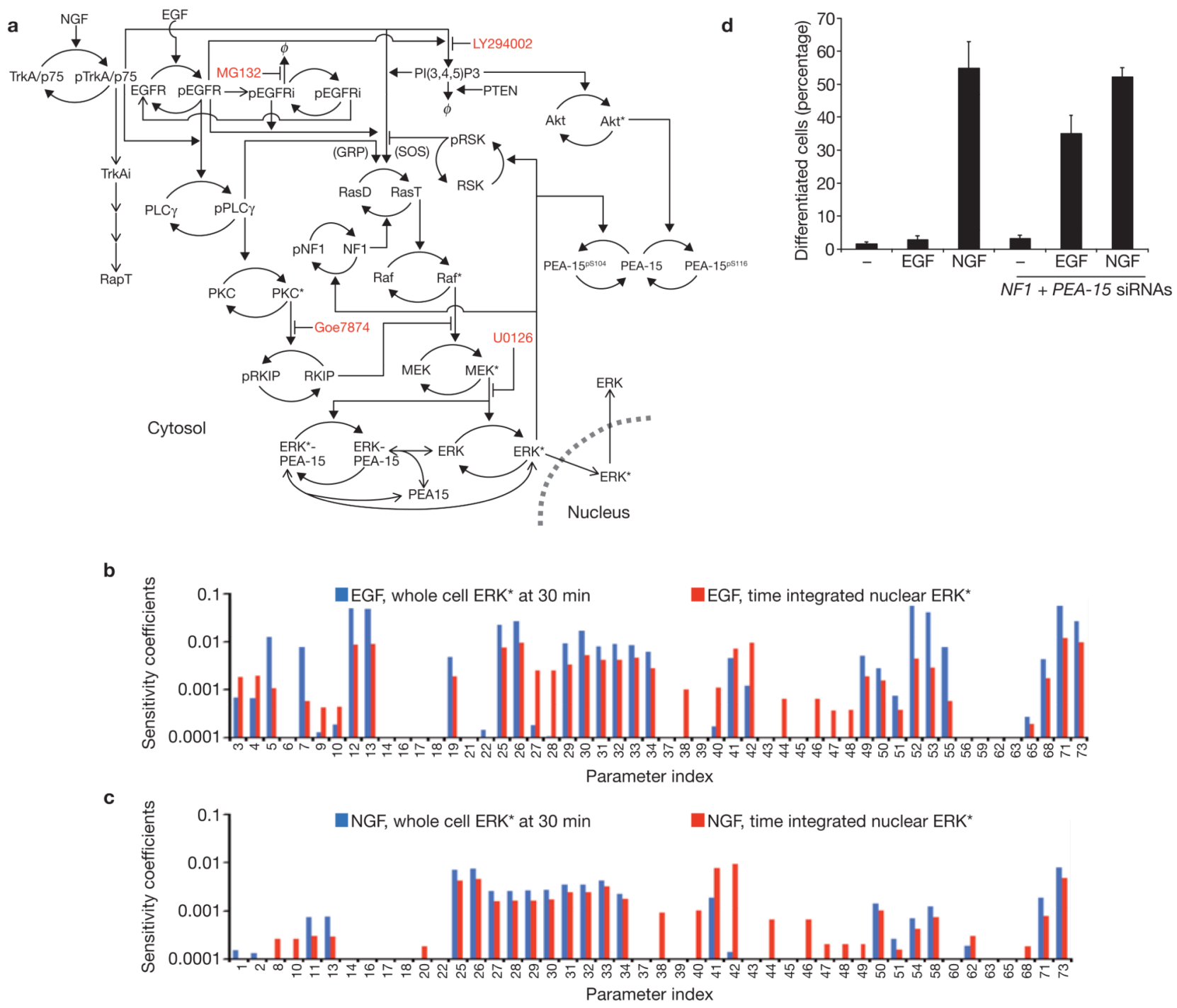

Figure 5.

Kinetic mathematical model of the ERK pathway in PC12 cells. (a) Topology scheme of the model. Asterisks indicate active proteins. (b, c) Sensitivity analysis of EGF (b) and NGF (c) treatment. Raw sensitivity coefficients were calculated by making $1 \%$ changes in model parameters, and then calculating the change in either the amount of whole cell ERK* at 30 min after stimulation, or the time integral of the nuclear ERK* profile. These raw coefficients were normalized by dividing by the baseline value (no parameter perturbation), and then absolute values were taken. (d) Cooperation between NF1- and PEA-15knockdown. Cells were co-transfected with an eGFP expression vector plus control or NF1 and $P E A-15$ siRNA. Serum-starved cells were treated with EGF or NGF for $24 \mathrm{~h}$, and scored for differentiation as in Fig. $2 \mathrm{~g}$. 